\title{
Intermediate outcomes of ab externo circumferential trabeculotomy and canaloplasty in POAG patients with prior incisional glaucoma surgery
}

\author{
Huaizhou Wang ${ }^{1 \dagger}$, Chen $\mathrm{Xin}^{1 \dagger}$, Ying Han ${ }^{2}$, Yan Shi', Sarah Ziaei ${ }^{2}$ and Ningli Wang ${ }^{1 *}$ (D)
}

\begin{abstract}
Background: To compare the efficacy and safety of ab externo circumferential trabeculotomy (ECT) and canaloplasty on primary open angle glaucoma (POAG) patients with failed filtering surgery and intact schlemm's canal (SC).

Methods: We conducted a retrospective chart review of POAG patients with failed filtering surgery and intact SC, who further received ECT and canaloplasty. The primary outcome measures were intraocular pressure (IOP) and the number of topical medications at each follow-up point. The secondary outcome compared the quantified success rate at1-year follow-up between the groups.

Results: Twenty-nine eyes were recruited in the ECT group and 19 eyes in canaloplasty group. The postoperative IOP and the number of topical medications decreased significantly in both groups $(p<0.001)$. The IOP at 3 -month and 6-monthwas significantly lower in the ECT group $(p=0.039, p=0.001)$ than in the canaloplasty group. Although the IOP at 12-mon was similar between the two groups, the number of topical medications was less in the ECT group ( $p=0.040)$. Hyphema $(72.4 \%)$ and ciliary body detachment $(27.6 \%)$, which mainly resolve spontaneously, were two leading complications in the ECT group. The prevalence of hyphema was higher in ECT than in canaloplasty group $(p<0.001)$.
\end{abstract}

Conclusion: For POAG with failed filtering surgery and intact SC, canaloplasty may be safer, whereas ECT presented better IOP control.

Translational Relevance: Suggestions for surgical choice for POAG with failed filtering surgery.

Keywords: Ab externo circumferential trabeculotomy, Canaloplasty, Primary open angle glaucoma, Failed filtering glaucoma surgery

\footnotetext{
* Correspondence: wningli@vip.163.com

${ }^{\dagger}$ Huaizhou Wang and Chen Xin contributed equally to this work.

${ }^{1}$ Beijing Tongren Eye center, Beijing Tongren Hospital, Capital medical university, Beijing, China

Full list of author information is available at the end of the article
}

(c) The Author(s). 2020 Open Access This article is licensed under a Creative Commons Attribution 4.0 International License, which permits use, sharing, adaptation, distribution and reproduction in any medium or format, as long as you give appropriate credit to the original author(s) and the source, provide a link to the Creative Commons licence, and indicate if changes were made. The images or other third party material in this article are included in the article's Creative Commons licence, unless indicated otherwise in a credit line to the material. If material is not included in the article's Creative Commons licence and your intended use is not permitted by statutory regulation or exceeds the permitted use, you will need to obtain permission directly from the copyright holder. To view a copy of this licence, visit http://creativecommons.org/licenses/by/4.0/. The Creative Commons Public Domain Dedication waiver (http://creativecommons.org/publicdomain/zero/1.0/) applies to the data made available in this article, unless otherwise stated in a credit line to the data. 


\section{Background}

Glaucoma is the leading cause of irreversible blindness worldwide and it is estimated that 65.5 millions of population will involve in primary open angle glaucoma (POAG) in 2020 [1]. Surgical intervention is needed when patients fail in conservative treatment. Filtering surgery, such as trabeculectomy, is one commonly performed approach. The fistula between the anterior chamber and subconjunctival bleb is crucial for its success. However, the fistula is also involved in postoperative complications. Eventual formation of scar, blocking the fistula, leads to the failure of the surgery [2, 3]. It was reported that the failure rate of glaucoma filtering surgery reached $46.9 \%$ by postoperative 5 -year follow-up and was even higher in patients with prior failed filtering surgery [4].

Bleb independent procedures, canaloplasty and ab externo circumferential trabeculotomy (ECT), were proposed this century and have become popular in clinic. It was reported that canaloplasty $[5,6]$ and ECT $[7,8]$ provide good IOP reduction with a favorable safety profile as an initial procedure for POAG. Moreover, canaloplasty is also a candidate for POAG with prior failed filtering surgeries with or without intact schlemm's canal (SC) $[9,10]$. Recently, a study reported that circumferential trabeculotomy could be a feasible remedy for POAG with failed incisional glaucoma surgery [11] and failed canaloplasty [12].

However, there were no studies that either directly compared the surgical outcome between canaloplasty and ECT or solely focused on the patient who had failed glaucoma filtering surgery. Here, we compared safety and efficacy of canaloplasty and ECT for POAG with failed glaucoma filtering surgery with 1-year follow-up.

\section{Methods}

A retrospective chart review was performed for all POAG patients with failed filtering surgery who received canaloplasty or ECT procedures to further control IOP in the glaucoma department of Beijing Tongren Hospital between Feb 1st, 2014 and Dec 31st, 2016. All surgical procedures were done by senior glaucoma specialists, Dr. WHZ and WNL. The study followed the tenants of the Declaration of Helsinki and was approved by the institutional ethics committee at Beijing Tongren Hospital.

Inclusion criteria for this study were: (1) POAG patients received failed glaucoma filtering surgery, presenting with either an IOP $>21 \mathrm{mmHg}$ or progressive deterioration of visual field defects on maximum tolerable medications. (2) Preoperative gonioscopic and ultrasound biomicroscopic examination revealed an open angle and identifiable intact Schlemm's canal (SC). (3) No visible filtering bleb exists. Exclusion criteria were: other types of glaucoma, POAG with failed incisional glaucoma surgery and iris synechia or broken SC.

The full details of the surgical procedures were reported previously $[9,13]$. Briefly, fornix-based conjunctival and scleral flaps were made next to the prior incision. Exposure of the lumen of SC was followed by the circumferential catheterization of SC. For canaloplasty, the viscoelastic (Healon GV, Abbott Vision, Abbott Park, IL, USA) was injected and 10-0 Polypropylene suture on tension was placed with the retraction of the microcatheter from the SC. If the circumferential catheterization could not be achieved, viscocanalostomy was done instead. For ECT, the two ends of the microcatheter were grasped and pulled in opposite directions. Thus, the trabecular meshwork (TM) was broken circumferentially. If the circumferential catheterization could not be achieved, TM was incised with Harms trabeculotome. Finally, the scleral and conjunctival flaps were sutured tightly in both groups.

After surgery, all subjects were given topical drops of combined tobramycin and dexamethasone for 2 weeks. The subjects receiving ECT were on topical 2\% pilocarpine three times per day for 3 months.

The primary outcomes included IOP and the number of glaucoma medications at postoperative 1-month, 3month, 6-month and 12-month follow-up visits. The secondary outcome was the success rate, which was defined as: (1) $\mathrm{IOP} \leq 21 \mathrm{mmHg}$ and a reduction in $\mathrm{IOP} \geq$ $20 \%$ from baseline, (2) no additional glaucoma surgery was required, and (3) patients with or without medication.

All statistical analyses were performed with SPSS 18.0. Preoperative and postoperative measurements were compared using ANOVA analysis and Tukey paired comparisons. Independent $t$ test was used to compare the IOP and medication between groups. Kaplan-Meier methods were used to calculate cumulative success rates for the two groups, which was compared with log-rank test. A $p<0.05$ was considered statistically significant.

\section{Results}

A total of 48 eyes in 45 patients were recruited, including 29 eyes (27 patients) in the ECT group and 19 eyes (18 patients) in the canaloplasty group. Demographics of the recruited patients are shown in Table 1 . The types of prior glaucoma filtering surgery included trabeculectomy, Ex-PRESS shunt implantation and deep sclerectomy. The number of failed glaucoma surgeries was $1.3 \pm$ 0.7 in the ECT group, including 6 eyes with 2 failed procedures and 1 eye with 3 failed procedures. The canaloplasty group had on average $1.3 \pm 0.4$ prior failed filtering surgeries, including 6 POAG eyes with 2 failed procedures. There is no significant difference between the two groups in either the number of prior surgeries 
Table 1 Patient Demographics

\begin{tabular}{llll}
\hline Parameters & ECT & Canaloplasty & $P$-value \\
\hline Number of eyes & 29 & 19 & \\
Sex & & & 0.805 \\
$\quad$ Male, n (\%) & $16(65.5 \%)$ & $10(52.6 \%)$ & \\
$\quad$ Female, n (\%) & $11(34.5 \%)$ & $8(47.3 \%)$ & \\
Side of eyes & & & 1.00 \\
$\quad$ Right, n (\%) & 15 & 9 & \\
$\quad$ Left, n (\%) & 14 & 10 & \\
Age, mean \pm SD (year) & $35.0 \pm 7.1$ & $40.1 \pm 12.9$ & 0.082 \\
Number of prior surgeries, & $1.3 \pm 0.7$ & $1.3 \pm 0.5$ & 0.787 \\
mean \pm SD & & & \\
Types of failed filtering surgeries & 41 & 25 & 0.214 \\
Trabeculectomy, n (\%) & $35 / 41$ & $18 / 25$ & 0.721 \\
Ex-PRESS shunt, n (\%) & $5 / 41$ & $4 / 25$ & 0.148 \\
Non-penetrating, n (\%) & $1 / 41$ & $3 / 25$ & 0.394 \\
\hline Intervals mean \pm SD (month) & $19.8 \pm 8.5$ & $17.7 \pm 8.0$ &
\end{tabular}

$E C T$ ab external circumferential trabeculotomy Intervals: The interval between the last prior incisional glaucoma surgery and the bleb independent procedure

$(p=0.787)$ or the interval between the last prior failed incisional glaucoma surgery and the bleb independent procedure $(p=0.394)$.

In the ECT group, 3 out of 29 eyes received TM incision with Harms trabeculotome. The postoperative IOPs were all significantly decreased from the baseline $(p<$ 0.01 ). The IOPs at 6 -mon and 12-mon were further decreased and significantly lower than IOPs at 1 -mon $(p=$ $0.029, p=0.022)$. The number of topical medications was significantly decreased from baseline $(p<0.001)$, and it decreased further in 6-mon and 12-mon than that in 1 -mon and 3-mon $(p=0.01, p<0.01)$.

In the canaloplasty group, except two with viscocanalostomy, 17 eyes received circumferential catheterization and tensioning suture placement. The postoperative IOPs were all significantly decreased from the baseline $(p<0.01)$, but were not significantly different from each other. The number of topical medications was significantly different from the baseline $(p<0.01)$, but there was no significant difference since 1-mon and afterwards.

In terms of comparison between groups, the postoperative IOPs at 3-mon and 6-mon were significantly lower in the ECT group than in the canaloplasty group (Table 2). The number of topical medications was similar between two groups at 1-mon, 3-mon and 6-mon postoperatively $(p=0.124, p=0.668$, and $p=0.053$ respectively), whereas at 12 -mon it was less in the ECT group than in the canaloplasty group $(p=0.040)$ (Table 3). The Kaplan-Meier survival analyses demonstrated that the one-year cumulative probabilities of
Table 2 Intraocular pressure (IOP) measurement (mmHg)

\begin{tabular}{clll}
\hline Parameters & ECT & Canaloplasty & $P$-value \\
\hline Baseline & $29.3 \pm 9.6$ & $29.5 \pm 7.9$ & 0.947 \\
Postoperative (month) & & \\
$1-$ & $19.0 \pm 7.9$ & $19.2 \pm 5.4$ & 0.896 \\
$3-$ & $16.5 \pm 3.5$ & $18.6 \pm 3.4$ & 0.039 \\
$6-$ & $15.2 \pm 3.4$ & $17.7 \pm 1.2$ & 0.001 \\
$12-$ & $15.0 \pm 2.5$ & $16.3 \pm 2.0$ & 0.071 \\
$P$ value & $<0.001$ & $<0.001$ & \\
\hline
\end{tabular}

success of ECT and canaloplasty was 86.2 and $84.2 \%$ respectively, which was not significantly different between groups ( $p=0.866)$ (Fig. 1).

In the ECT group, all the patients presented intraoperative blood reflux into the anterior chamber. On day 1 , all the patients demonstrated hyphema in different degrees, from scattered floating blood cells to $6 \mathrm{~mm}$ hyphema. The hyphema in 21 eyes (72.4\%) resolved spontaneously in 7 days with no significant IOP increase. Among them,8 patients (27.6\%) demonstrated ciliary detachment in anterior segment optical coherent tomography, which resolved spontaneously and did not lead to hypotony. Seven subjects experienced IOP spike during the postoperative 2 weeks. Among them were 6 patients on topical medication for whom IOP gradually decreased, and the medication was stopped within 2 weeks. Another patient received anterior chamber irrigation once. One patient presented hypotony and ciliary detachment for 7 days, and topical 2\% pilocarpine was held until ciliary detachment resolved spontaneously.

In canaloplasty group, one subject presented hyphema with scattered blood cells (5.3\%), which resolved spontaneously in 3 days. None experienced IOP spike within 2 weeks after surgery. One subject presented hypotony and ciliary detachment (5.3\%) that resolved spontaneously in 1 week.

\section{Discussion}

Repeated glaucoma filtering surgery in POAG patients leads to higher failure rate and complications. SC-based

Table 3 Number of topical medications ( $n$ )

\begin{tabular}{llll}
\hline Parameters & ECT & Canaloplasty & $P$-value \\
\hline Baseline & $3.2 \pm 0.6$ & $2.8 \pm 0.9$ & 0.089 \\
Postoperative (month) & & \\
$1-$ & $1.7 \pm 2.0$ & $0.9 \pm 1.2$ & 0.124 \\
$3-$ & $1.0 \pm 0.9$ & $0.9 \pm 1.2$ & 0.668 \\
$6-$ & $0.3 \pm 0.7$ & $0.9 \pm 1.1$ & 0.053 \\
$12-$ & $0.3 \pm 0.6$ & $0.8 \pm 1.0$ & 0.040 \\
$P$ value & $<0.001$ & $<0.001$ & \\
\hline
\end{tabular}




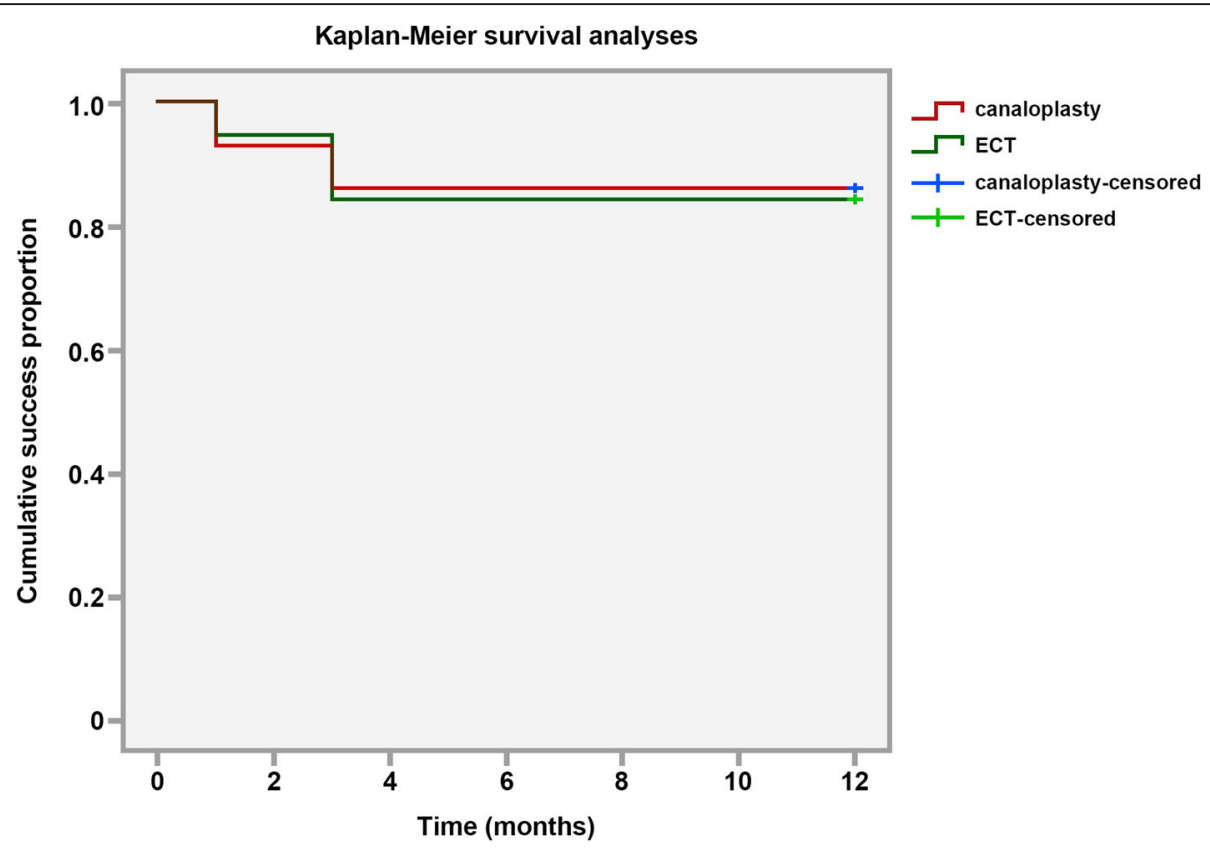

Fig. 1 The cumulative probabilities of success of ECT and canaloplasty

surgical procedures, devoid of bleb, presented good safety and efficacy and are probably of potential benefit to the POAG patient with failed glaucoma filtering surgery. Canaloplasty and ECT are two representative $\mathrm{Ab}$ externo procedures of SC-based glaucoma surgery. Canaloplasty was reported to be a feasible, efficient, and safe candidate for POAG with prior incisional glaucoma surgery with both intact and disrupted SC $[9,10]$. Circumferential trabeculotomy, either ab interno or ab externo, was also practiced on POAG patients and presented good IOP control [7, 8]. Recently, a study reported that gonioscopy-assistant transluminal trabeculotomy appears to be safe and successful in treating 60 to $70 \%$ of POAG patients with prior incisional glaucoma surgery [11]. Meanwhile, some studies reported that the ab interno 360-degree suture trabeculotomy is a simple and safe technique to further enhance the IOP-lowering and drugsparing effect of canaloplasty [14].

In this study, the circumferential catheterization of canal was achieved in 89.7 and $89.5 \%$ patients in ECT group and canaloplasty group. It was reported that besides the POAG-related changes in TM, continuous bypass of aqueous humor from the TM pathway further leads to more subendothelial deposits in TM and the amorphous material piling up in juxtacanulicular tissue. In turn, this leads to deterioration and collapse of the canal and decreased potential drainage ability $[15,16]$. Unlike common blood vessels, the unique lymphatic-like phenotype of SC endothelium provides the potential lumen and recovery of the drainage even when it is collapsed for a long time, which remains the opportunity of the MIGS $[17,18]$.
Our study presented the similar 1-year cumulative quantified success rate of ECT and canaloplasty. In general, the postoperative IOP and the number of topical medications significantly decreased after either ECT or canaloplasty procedure. Although the subjects with ECT received pilocarpine continuously for 3 months in prevention of the scarring of the cut-edge along TM and the TM-iris adhesion, the POAG with ECT received a similar number of topical medications as canaloplasty at 1-mon, 3-mon and 6-mon. However, the postoperative IOP is lower in ECT at 3-mon and 6-mon than that in the canaloplasty group. At 12-mon, IOP were comparable for subjects with ECT and canaloplasty, but the number of topical medications in ECT group was lower than that in the canaloplasty group. These results implied that ECT may be relatively more efficient in IOP control than canaloplasty for the subjects recruited in this study, who had an average IOP of $30 \mathrm{mmHg}$ with maximal topical medications. Previous study on normal cadaver human eyes showed that considering the initial IOP of $30 \mathrm{mmHg}$, the six-hour trabeculotomy or threehour sinusotomy could proximately decrease $65 \%$ or $50 \%$ resistance of aqueous outflow [19]. In eyes with glaucoma, the resistance generated from TM dominated [20]. The ECT, probably eliminating all the resistance in the TM region, presents quick and good IOP control. Comparatively, canaloplasty reduces the IOP possibly by the mechanism of trans-descent window drainage, dilation of the SC and surrounding the collector channel (CC), recovery of the TM herniation in $\mathrm{CC}$, disruption of the $\mathrm{SC}$ endothelium, and creation of more pores and 
cell junction detachment [21]. Thus, it directly decreased the resistance at the region of exposure of the SC and descent window, which is relatively less efficient than the circumferential trabeculotomy. However, it probably takes some time for regeneration of the drainage of the aqueous humor.

In terms of complications, canaloplasty seems to be safer. Canaloplasty did not induce any IOP spike or complication that needed to be resolved by further surgical procedure. Hyphema is more common and severer in the ECT group. Hypotony and ciliary body detachment is more frequent in the ECT group as well. Large fluctuation of IOP during the ECT procedure and repeated paracentesis could lead to ciliary body detachment [22]. Besides this, the potential for exacerbation of postoperative inflammation induced by the application of topical pilocarpine might be another reason for the ciliary body detachment and persistent hypotony [23]. Potential reasons for the IOP fluctuation in ECT patients include the recovery of the hypotony and ciliary body detachment and the aqueous drainage decrease in the uveaoscleral pathway induced by the topical pilocarpine [24].

Although it is proposed that topical pilocarpine could prevent the scarring of the TM cut-edge and the TMiris adhesion, its potential effect on contraction of ciliary body and breakdown of the aqueous-blood barrier, which might increase failure of the ECT in the long run, warrant attention.

Repeated ab externo procedures and multiple scleral flaps may lead to substantial damage to the distal aqueous pathway, which is more severe in POAG with prior incisional surgeries. With newer surgical approaches, such as the gonioscopy assisted transluminal trabeculotomy (GATT), conjunctiva and scleral territory would be better preserved. This could lead to even better results for the repeat Schlemm's canal surgery because of the preserved distal aqueous pathway.

The current study has several limitations. First, our sample size was small with one-year follow up. Second, the study was a retrospective study, although both groups were comparable. A randomized clinical trial should be performed to better understandthe efficacy of ECT and canaloplasty for POAG.

\section{Conclusion}

In conclusion, for POAG with prior incisional glaucoma surgery, both canaloplasty and ECP decreased postoperative IOP and number of medications with high success rate at one-year follow up. While canaloplasty seems relatively safe with less postoperative complications, whereas ECT may achievebetter IOP control with fewer number of topical medications.

\section{Abbreviations}

ECT: Externo circumferential trabeculotomy; POAG: Primary open angle glaucoma; SC: Schlemm's canal; IOP: Intraocular pressure; TM: Trabecular meshwork; CC: Collector channel; GATT: Gonioscopy assisted transluminal trabeculotomy

\section{Acknowledgements}

Not applicable.

\section{Authors' contributions}

WHZ and XC collected and analyzed patient data and wrote the article. WHZ performed some of the canaloplasty and ECT. They contributed equally and should therefore be regarded as co-first authors. HY was one of major contributor to designing the research, SY collected information from the published literature and helped to collect the patient data. ZS contributed in writing the article. WNL was the corresponding author who designed the research and performed most of the surgeries. All authors read and approved the final manuscript.

\section{Funding}

This study was supported by Beijing Municipal Administration of Hospitals' Youth Programme. (QML20180202,) from Chen Xin who was the co-first author and National Natural Science Foundation of China (U1713220) from Ningli Wang who was the corresponding author.

\section{Availability of data and materials}

The datasets used and analyzed during the current study are available from the corresponding author upon reasonable request.

Ethics approval and consent to participate

The study followed the tenants of the Declaration of Helsinki and was approved by the institutional ethics committee at Beijing Tongren Hospital.

Consent for publication

Not Applicable.

\section{Competing interests}

The authors declare that they have no competing interests.

\section{Author details}

${ }^{1}$ Beijing Tongren Eye center, Beijing Tongren Hospital, Capital medical university, Beijing, China. ${ }^{2}$ Department of Ophthalmology, University of California, San Francisco School of Medicine, San Francisco, California, USA.

Received: 14 May 2020 Accepted: 14 September 2020

Published online: 02 October 2020

\section{References}

1. Song P, Wang J, Bucan K, Theodoratou E, Rudan I, Chan KY. National and subnational prevalence and burden of glaucoma in China: a systematic analysis. J Glob Health. 2017;7(2):020705.

2. Gedde SJ, Schiffman JC, Feuer WJ, et al. Treatment outcomes in the tube versus trabeculectomy (TVT) study after five years of follow-up. Am J Ophthalmlol. 2012;153:789-803.e2.

3. Buys YM. Trabeculectomy with ExPRESS: weighing the benefits and cost. Curr Opin Ophthalmol. 2013;24:111-8.

4. Rulli E, Biagioli E, Riva l, et al. Efficacy and safety of trabeculectomy vs. nonpenetrating surgical procedures: a systematic review and meta-analysis. JAMA Ophthalmol. 2013;131:1573-82.

5. Khaimi MA, Dvorak JD, Ding K. An analysis of 3-year outcomes following Canaloplasty for the treatment of open-angle glaucoma. J Ophthalmol. 2017;2017:2904272.

6. Riva I, Brusini P, Oddone F, et al. Canaloplasty in the treatment of openangle glaucoma: a review of patient selection and outcomes. Adv Ther. 2019;36(1):31-43.

7. Yalinbas D, Dilekmen N, Hepsen IF. Comparison of ab externo and ab interno 360-degree suture Trabeculotomy in adult open-angle glaucoma. J Glaucoma. 2020;1:1.

8. Grover DS, Godfrey DG, Smith O, et al. Gonioscopy-assisted transluminal trabeculotomy, ab interno trabeculotomy: technique report and preliminary results. Ophthalmology. 2014;121(4):855-61. 
9. Xin C, Chen X, Shi Y, et al. One-year interim comparison of canaloplasty in primary open-angle glaucoma following failed filtering surgery with primary canaloplasty. Br J Ophthalmol. 2016;100(12):1692-6.

10. Xin C, Chen X, Shi Y, et al. Modified Canaloplasty: a new, effective, and safe option for glaucoma patients with a disrupted Schlemm Canal wall. J Glaucoma. 2016;25(10):798-801.

11. Grover DS, Godfrey DG, Smith O, et al. Outcomes of Gonioscopy-assisted Transluminal Trabeculotomy (GATT) in eyes with prior incisional glaucoma surgery. J Glaucoma. 2017;26(1):41-5.

12. Baumgarten $S$, Kürten $D$, Lohmann $T$, et al. Outcomes of $360^{\circ}$ suture trabeculotomy after unsuccessful canaloplasty. Graefes Arch Clin Exp Ophthalmol. 2020;258(2):387.

13. Shi Y, Wang H, Yin J, et al. Outcomes of microcatheter-assisted trabeculotomy following failed angle surgeries in primary congenital glaucoma. Eye. 2017;31:132-9.

14. Voykov B, Szurman P, Dimopoulos S, et al. Micro-invasive suture trabeculotomy after canaloplasty: preliminary results. Clin Exp Ophthalmol. 2015;43(5):409-14.

15. Ito S, Nishikawa M, Tokura T, et al. Histopathological study of trabecularmeshwork after trabeculotomy in monkeys. Nippon Ganka Gakkai Zasshi. 1994;98:811-9.

16. Johnson DH, Matsumoto Y. Schlemm's canal becomes smaller after successfulfiltration surgery. Arch Ophthalmol. 2000;118:1251-6.

17. Ethier CR. The inner wall of Schlemm's canal. Exp Eye Res. 2002;74:161-72.

18. Truong TN, Li H, Hong YK, et al. Novel characterization and live imaging ofSchlemm's canal expressing Prox-1. PLoS One. 2014:9:e98245.

19. Ellingsen BA, Grant WM. Trabeculotomy and sinusotomy in enucleated human eyes. Investig Ophthalmol. 1972;11:21-8.

20. Johnstone M. And Grant Morton. Microsurgery of schlemm's canal and the human aqueous outflow system. Am J Ophthalmol. 1973;76(3):906-17.

21. Xin C, Tian N, Li M, et al. Mechanism of the reconstruction of aqueous outflow drainage.Sci China. Life Sci. 2018;61(5):534-40.

22. Brubaker RF, Pederson JE. Ciliochoroidal detachment. Surv Ophthalmol. 1983;27(5):281-9.

23. Baudouin C. Mechanisms of failure in glaucoma filtering surgery: a consequence of antiglaucomatousdrugs? Int J Clin Pharmacol Res. 1996; 16(1):29-41.

24. Toris CB, Alm A, Camras CB. Latanoprost and cholinergic agonists in combination. Surv Ophthalmol. 2002:47(Suppl 1):S141-7.

\section{Publisher's Note}

Springer Nature remains neutral with regard to jurisdictional claims in published maps and institutional affiliations.

Ready to submit your research? Choose BMC and benefit from:

- fast, convenient online submission

- thorough peer review by experienced researchers in your field

- rapid publication on acceptance

- support for research data, including large and complex data types

- gold Open Access which fosters wider collaboration and increased citations

- maximum visibility for your research: over $100 \mathrm{M}$ website views per year

At BMC, research is always in progress.

Learn more biomedcentral.com/submissions 